\title{
Comparison of Topology Based-Routing Protocols in Wireless Network
}

\author{
Vikas Sharma ${ }^{1}$, Anita Ganpati ${ }^{2 *}$
}

\begin{abstract}
VANET (Vehicular Ad-hoc Network) is a mobile Ad-hoc Network which deals with the moving vehicles. VANET supports Intelligent Transport Systems (ITS) which is related to different modes of transport and traffic management techniques. VANETs enabled users to be informed and make them safer. VANET uses IEEE 802.11p standard wireless access protocol for communication. An important and necessary issue of VANET is to design routing protocols. In a network, communication takes place by the use of the routing protocols. There are mainly two types of communications used such as Vehicle to Vehicle (V2V) and Vehicle to Infrastructure (V2I) in VANET. Vehicles can send and receive messages among them and also to and from infrastructure used. In this paper, AODV, DSR and DSDV are compared by analysing the results of simulation on various metrics such as average throughput, instant throughput, packet delivery ratio and residual energy. Findings indicates utilization of AODV and DSR is more applicable for these metrics as compared to DSDV. A network simulator (NS2) is used for simulation.
\end{abstract}

Key Words: AODV (Ad hoc On-Demand Distance Vector), DSR (Dynamic Source Routing), DSDV (Destination-Sequenced DistanceVector), NS2 (Network Simulator 2), VANET (Vehicular Ad-hoc Network).

\section{INTRODUCTION}

In today's world, Roadway transportation is congested because of the high usage of vehicles and less knowledge of traffic rules. Unsafe speeds, bad weather conditions and heavy traffic may lead to accidents and loss of life. More than $60 \%$ accidents take place owed to the delay of less than half second. These kinds of problems are avoidable by introducing the Vehicular Ad-hoc Network. A network which doesn't have a pre-existing communication infrastructure is Vehicular Ad-hoc Network (VANET). Network is created by some nodes which are available in surroundings. So, the determination of nodes i.e. from which node to transfer data and to whom is done dynamically, depending upon the connectivity of both devices. Ad-hoc networks can use flooding for data transfer among the nodes. In Ad-hoc network, all devices are treated equally and also have same status. Vehicular Ad-hoc Network is a mobile ad hoc network which is concerned with moving vehicles called as nodes. In VANET, messages are transferred to each other by the node or router. VANETs come up with advanced vehicles fitted with GPS and Wi-Fi devices. These outfits are important to communicate with each other within the networks range. There are two varieties of communications already presented in VANET one is Vehicle-to-Vehicle (V2V) and another is Vehicle-toInfrastructure (V2I) also sometimes referred to as Vehicle to Roadside unit (V2R) [1]. In V2V communication vehicles send and receive messages to and from each other. These messages may be coverage of road congestion, accidents ahead, etc. which in turns may avoid an accident or other loss. V2I communications are among nodes and road aspect infrastructure, for e.g. covering of an incident or a malicious node, helps in finding nearest petrol station, on-line toll payment, etc. Usually, VANET is used to evade the traffic and driving problems i.e. change in location constantly. To simulate VANET we have to know about few tools like NS-2, Omnet++, and etc. [2].

\section{ROUTING PROTOCOLS}

A process of moving information across internet from source to destination is called as routing. There are various protocols used in routing. As VANET have dynamic nature of topology, this mechanism provides an optimal path between network nodes by reducing the overhead [3], [4]. Routing protocols are classified into two main categories i.e. Topology Based Routing Protocols and Position Based Routing Protocols.

\footnotetext{
Manuscript received April26, 2019; Revised June05; Accepted June 08, 2019. (ID NoJMIS-19M-04-014)

Corresponding Author (*): Antia Ganpati, Department of Computer Science, Himachal Pradesh University, Shimla, India

1,2 Department of Computer Science, Himachal Pradesh University, Shimla, India, Vssharma213@gmail.com, anitaganpati@gmail.com 2
} 
Topology based routing protocol is traditional VANET routing protocol. It uses source to destination information that is stored in routing table [5]. Whereas, Position based routing or geographic routing is based on the positional information of nodes in routing process. For utilizing the source node, it sends a packet to the destination node using geographic position of individual node. AODV is reactive (on-demand) protocol which is proposed for Mobile ad hoc Network. Packet headers are not included for routes of AODV and are highly dynamic in nature which reduces overhead [6]. The information used for routing is stored in source node, destination node and intermediate nodes along with active routing in transmission of data. AODV for both unicasts and multicasts uses the flag in the packets [10]. DSDV is a type of table-driven protocol. This protocol provides loop free routing and by utilizing the frequent updates in routing table it decreases the extra traffic. This also reduces routing overhead and always choose optimal path by using shortest path algorithm [7]. DSDV provides the sequence number to avoid the duplication entry into the routing table. Extra traffic is avoided with incremental updates instead of full dumped updates. This algorithm is suitable for small Ad-hoc networks [8]. DSR provide a high on-demand routing process which has low overhead protocol and fast reaction on the frequent changes in network topology. DSR protocol provides successful data packet delivery and allows multi hop routing in dynamic nature of network [9]. Two main processes in this protocol are: Route discovery and Route maintenance. When source node needs to communicate with node whose path is unavailable then the source node starts up a route discovery process in which the source node broadcasts route request messages to network. The destination node on receiving a RREQ packet, it sends back a route reply message to source node. Source node keeps the route in route cache for future communication. On the other hand, if routing fails, it sends back a route error to the source node with error message. In DSR, source node deletes path in cache and then store alternative path for destination [11].

\section{RELATED WORK}

A spotlight on various existing approaches for secure Ad Hoc routing process and their applicability to VANET'S and offer a wide range of applications improving road safety and driving comfort. The unique characteristics of VANETs compared to conventional mobile ad hoc networks and sensor networks pose particular challenges for a security solution for vehicular environment [3]. A system to analyze the vehicle density, data drop, throughput and end to end delay for AODV, DSDV and DSR routing protocols was proposed. Also, contrary to the pessimistic conclusion of previos works in done. Also, the analysation of the simulations which are performed to compare the performance of On-Demand and Table-Driven routing protocols by using the two different scenarios [5]. An investigation of different routing schemes that have been developed for VANETs, as well as by providing classifications of VANET routing protocols. Also, gives summarized comparisons between different classes in the context of their methodologies used, strengths, and limitations of each class scheme compared to other classes [6]. An efficient DSDV protocol for Ad Hoc Networks that overcome the problem of routes, and thereby improves the performance of regular DSDV. The proposed protocol has been implemented in the NS Simulator and performance comparison has been made with regular DSDV and DSR protocols [7]. The performance of AODV, DSDV and DSR was evaluated on the basis of various matrices for cluster based VANET and attempts are made to compare AODV, DSR and DSDV for various metrics [9]. A discussion on the characteristics and disadvantages of AODV, DSR and DSDV on the basis of difference of average end to end delay. The simulation is based on NS2 for these comparisons and founded that DSDV routing protocol has a poor performance in a largescale network with a variable structure as in the experiment of this research [10]. A review of AODV and DSDV conventions by displaying their qualities, usefulness, advantages and restrictions and after that makes their similar investigation so to examine their execution. The goal is to mention objective facts on how the execution of this protocol is able to move forward [11].

\section{SIMULATION AND PARAMETERS USED}

Research methodology used NS2, NSG and Gnuplot to obtain realistic results on different parameters. Here, parameters used are average throughput, instant throughput, packet delivery ratio and residual energy. NS2 is a network simulator which is used to simulate various routing protocols. NS2 consist all of common IP protocols and network animator (NAM) which is used to visualize the simulation. NSG is a network scenario generator which is a java-based tool that can run on any platform. Gnuplot is used for graphical representation of results. Fig.1 shows the communication between vehicles using Network Animator (NAM). NS2 uses TCL as its scripting language and simulate wired and wireless networks. NS2 uses OTcl to create and configure a network and use $\mathrm{C}++$ to run the simulation process. The $\mathrm{C}++$ defines the internal mechanism (i.e., a backend) of the simulation objects, the OTcl sets up simulation by assembling and configuring the objects as well as scheduling discrete events. TclCL is used to link the $\mathrm{C}++$ and OTcl. 


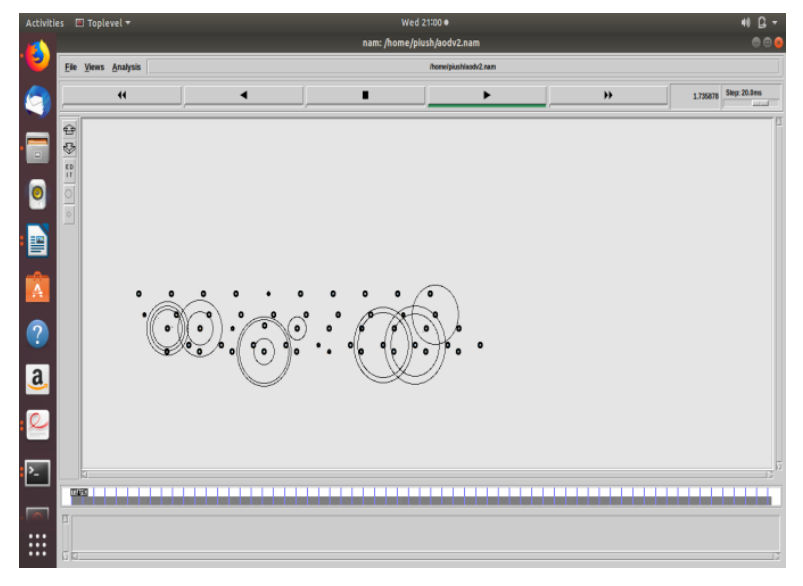

Fig. 1. V2V Communication.

In Fig.1, black dot represents the nodes used and circles represented the communication between vehicles. This representation is obtained with the help of NAM. The various simulation parameters are mentioned in Table 1. The maximum simulation time is 150 seconds for each case where nodes are varying and maximum speed is $30 \mathrm{~m} / \mathrm{s}$. omnidirectional antenna is used for transmission of data packet having size of 512 bytes. Throughput is defined as the average number of successfully delivered data packets. The result of throughput is calculated in kbps. Packet delivery ratio is the number of data packets which are successfully delivered to destinations by the number data packets that were sent by sources in the network. The energy remains in the nodes is referred as residual energy and is calculated as the difference of initial energy and energy consumed by the node. In this paper, evaluation on various performance parameters such as average throughput, instant throughput, packet delivery ratio and residual energy to compare AODV, DSR and DSDV routing protocols for varying number of vehicles is performed.

\section{RESULTS AND ANALYSIS}

To evaluate the different performance metrics mentioned different simulations are carried out to obtain results on different stages. The parameters used to analyze results are shown in Table 1 on which simulation is done. Fig. 2 shows the average throughput for AODV, DSR and DSDV v/s number of nodes. Fig.2 says that DSR has highest average throughput as compared to AODV and DSDV in case of different number of nodes.

Instant throughput for AODV, DSR and DSDV v/s number of nodes is shown in Fig.3. AODV has better results than DSR and DSDV for instant throughput measured in kbps for each case. DSR has average results produced during the simulation process. Whereas, DSDV produces some results where number of nodes are less which indicates that DSDV is not suitable for long range networks.

Table 1. Simulation Parameters.

\begin{tabular}{|c|c|}
\hline Network Simulator & Ns 2.35 \\
\hline Simulation Time & $150 \mathrm{~s}$ \\
\hline Routing Protocols & AODV, DSR, DSDV \\
\hline Traffic Type & TCP \\
\hline MAC Protocol & IEEE 802.11 \\
\hline Antenna Model & Omni Directional Antenna \\
\hline Application & FTP \\
\hline Number of Vehicles & $14,24,50$ \\
\hline Maximum Speed of Nodes & $30 \mathrm{~m} / \mathrm{s}$ \\
\hline Radio Propagation Model & Two Ray Ground \\
\hline Data Packet Size & 512 bytes \\
\hline Rate & $11 \mathrm{mb} / \mathrm{s}$ \\
\hline
\end{tabular}

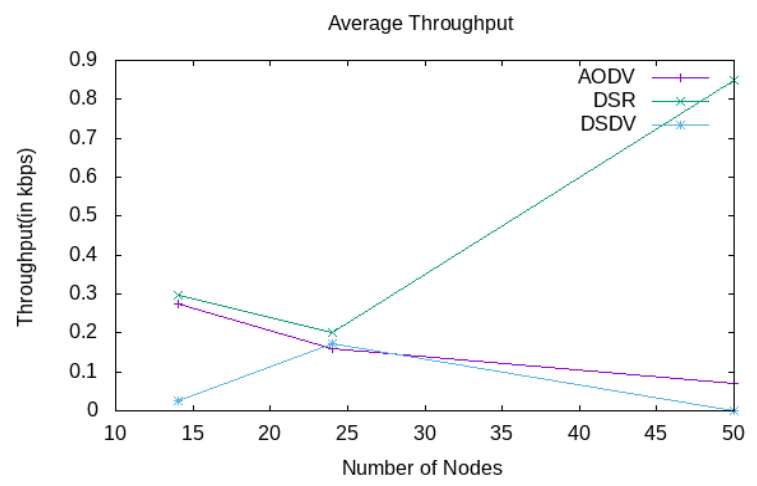

Fig. 2. Throughput v/s Number of nodes.

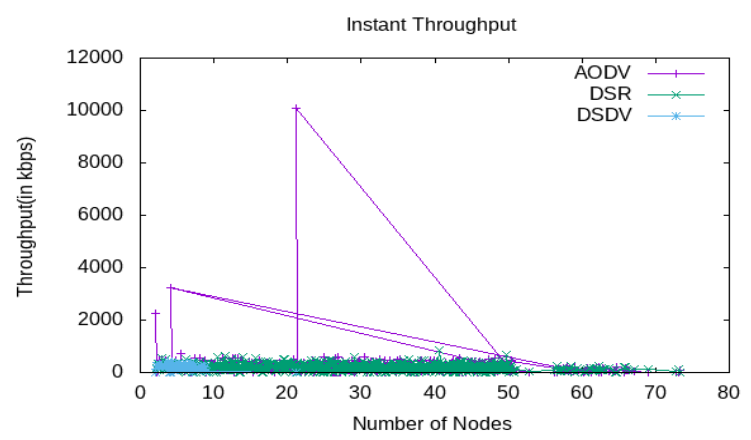

Fig. 3. Instant Throughput v/s Number of nodes. 


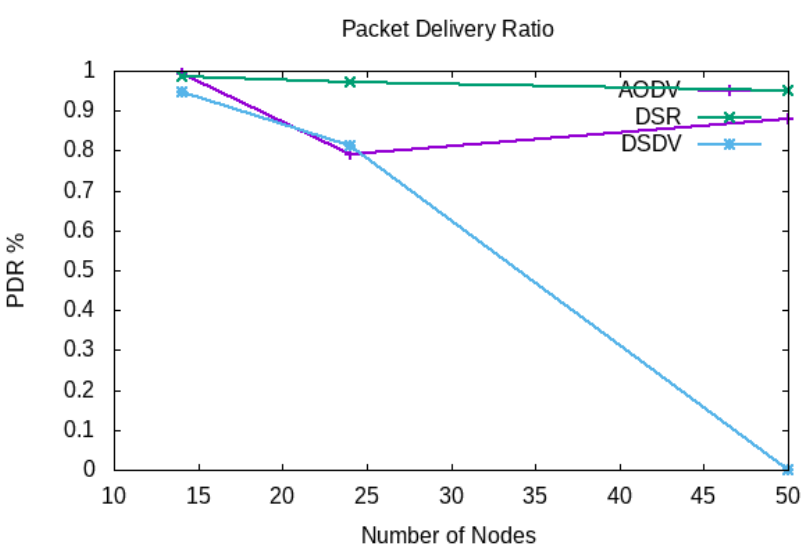

Fig. 4. Packet Delivery Ratio v/s Number of nodes.

Fig.4 shows the packet delivery ratio for AODV, DSR and DSDV v/s number of nodes. DSR having highest packet delivery ratio than AODV and DSR. AODV also have average packet delivery ratio as shown in Fig.4.

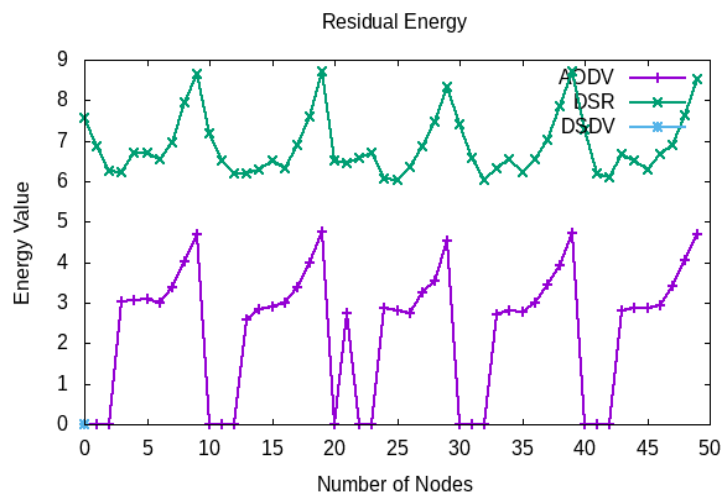

Fig. 5. Residual Energy v/s Number of nodes.

The results for residual energy for AODV, DSR and DSDV v/s Number of nodes taken in various stages of simulation process based on different parameters as discussed in Table 1. Some nodes in DSR maintain their energy through entire simulation process as shown in Fig.5.

\section{CONCLUSION}

The main goal of this research is to analyze and evaluate topology-based routing protocols in VANET. Here, analyzation for the performance of AODV, DSR and DSDV is done on parameters average throughput, instant throughput, packet delivery ratio and residual energy and results are based on the simulation done on various stages. These performances are measured with a network simulator ns2 under varying number of vehicles. On the basis of results obtained for average throughput, DSR can perform well when the number of nodes is minimum as compared to AODV and DSDV. AODV is good for the environment where number of nodes are maximum. Whereas, AODV having higher instant throughput. Also, DSR is better than AODV and DSDV in packet delivery
Comparison of Topology Based-Routing Protocols in Wireless Network

ratio. Packet delivery ratio of AODV is higher in case of minimum number of nodes. In the case of residual energy, in DSDV nodes has lost all their energy as compared to AODV and DSR and DSR have maximum nodes which do not lose their energy during simulation. The variation of results in DSDV is very low in four performance parameters and has a poor performance in a large-scale network. In this study, we have examined that how these protocols suffered from the dynamic nature of VANET.

\section{REFERENCES}

[1] C. Stergiou, K. E. Psannis, A. P. Plageras, Y. Ishibashi, B.-G. Kim, "Algorithms for efficient digital media transmission over IoT and cloud networking," Journal of Multimedia Information System, vol. 5, no. 1, pp. 110, March 2018.

[2] Vaesileios Memos, Kostas E. Psannis, Yutaka Ishibashi, Byung-Gyu Kim, Brij Gupta, “An Efficient Algorithm for Media-based Surveillance System (EAMSuS) in IoT Smart City Framework," Future Generation Computer Systems, Vol. 83, pp. 619-628, June 2017.

[3] A. P. Plageras, K. E. Psannis, C. Stergiou, H. Wang, B. B. Gupta, "Efficient IoT-based sensor BIG Data collection-processing and analysis in Smart Buildings," Future Generation Computer Systems, Vol. 82, pp. 349-357, May 2018.

[4] Emanuel Fonseca and Andreas Festag "A Survey of Existing Approaches for Secure Ad hoc Routine and their Applicability to VANET," NEC Network Laboratory, Vol. 28, PP 1-28, 2006.

[5] C. Stergiou, K. E. Psannis, B. B. Gupta "Advanced Media-based Smart Big Data on Intelligent Cloud Systems," IEEE Transaction on Sustainable Computing, in Press, 2018.

[6] MarwaAltayeb and ImadMahgoub "A Survey of Vehicular Ad hoc Networks Routing Protocols," International Journal of Innovation and Applied Studies, Vol. 3 No.3, 2013 PP 829-846.

[7] T Sri Harsha, Khaleel Ur Rahman Khan, A Venugopal Reddy, Rafi U Zaman and K. Aditya Reddy "An efficient DSDV Routing Protocol for Wireless Mobile Ad hoc Networks and Its Performance Comparison," IEEE, Vol. 4 No. 8, 2008 PP 506-511.

[8] S. El khediri "Survey of Syn-chronization Algorithms: Wireless Sensors Networks Case Study," in The Second International Conference on Communications and Information Technology (ICCIT), Vol.5 No.12, 2012.

[9] Yatendra Mohan Sharma and Saurabh Mukherjee "Comparative Performance Exploration Of AODV, DSDV \& DSR Routing Protocol In Cluster Based 
VANET Environment," International Journal of Advances in Engineering \& Technology, Vol. 4 No. 2, 2012 PP 120-127

[10] Prashant Kumar Maurya, Gaurav Sharma, VaishaliSahu, Ashish Roberts and Mahendra Srivastava "An Overview of AODV Routing Protocol," International Journal of Modern Engineering Research, Vol. 2 No.3, 2012 PP 728-732.

[11] C.Perkins "Highly Dynamic Destination-Sequenced Distance-Vector (DSDV) for Mobile Computer," InProc. of the ACM SIGCOMM, Vol. 24 No. 4, 1994 PP 234-244.

\section{Authors}

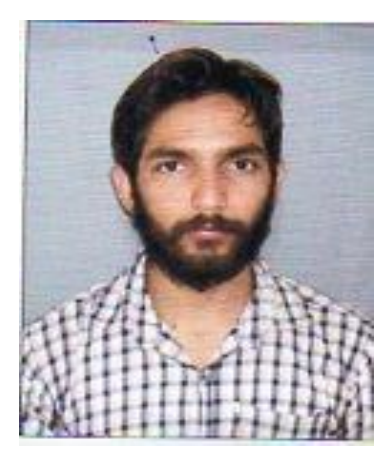

Vikas Sharma received his Bachelor's and Master's degree in Computer Applications from Himachal Pradesh University Shimla, India, in 2012 and 2016 respectively. In 2016, he joined the department of Computer Science for pursuing his M.Tech degree at Himachal Pradesh University Shimla, India.

algorithms used in wireless networks.

His research interests include various routing protocols and

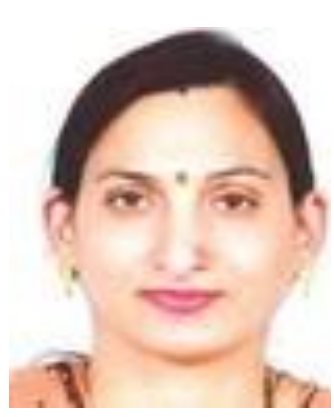

Anita Ganpati received her $\mathrm{PhD}$ degree in the Department of Computer Science from Himachal Pradesh University Shimla, India, in 2013. In 2000, she received an M.Tech (CSE) degree from Punjabi University Patiala, India and an Master's of Computer Application degree from Punjab University Chandigarh, India in 1997. She has seventeen years of teaching experience and presently working as Professor in the Department of Computer Science, Himachal Pradesh University Shimla, India.

She has published over 70 international journal and conference papers. Her research interests include Software Engineering, Open Source Software and Data Optimization. 
